\title{
Development of the Deoxycorticosterone Acetate (DOCA)-salt Hypertensive Rat Model
}

\author{
Nayara Pestana-Oliveira*, David B. Nahey, Tim Johnson and John P. Collister
}

Department of Veterinary and Biomedical Sciences, College of Veterinary Medicine, University of Minnesota, Saint Paul, MN 55108, USA

*For correspondence: pesta016@umn.edu

[Abstract] According to the World Health Organization (WHO), nearly 1.13 billion people worldwide have hypertension, a major factor responsible for premature death globally. The inherent multifactorial nature of hypertension makes its study difficult since the chronic rise in blood pressure depends on the intricate connection between dietary, genetic and environmental factors. Therefore, the pathophysiology of hypertension is not completely understood. For these reasons, there is an ongoing search for animal models that better mimic changes resulting from this disease. Because of its complexity, the use of animal models aimed at elucidating the pathogenesis of hypertension and to evaluate new therapeutic possibilities is an important tool for understanding this disease since it enables consistent experimental strategies that are impractical in humans. Over time, many animal models have been developed for the study of chronic increases in blood pressure ranging from genetic models that include the spontaneously hypertensive rat (SHR) and genetic manipulations, such as the TGR (mRen2) rat, as well as neurogenic or endocrine models. One of the most commonly used hypertensive rat models today is that of hypertension induced by treatment with deoxycorticosterone acetate associated with high sodium intake, i.e., the DOCA-salt model. This model is known to have a neurogenic component linked to increased sympathetic nervous system activity, and as such the DOCA-salt model promotes cross-talk between endocrine and neural components that lead to increased blood pressure, and may impact the functioning of other organs.

Keywords: DOCA-salt, Blood pressure, Neurogenic hypertension, High salt diet, Sympathetic nervous system

[Background] Hypertension is a chronic disease with the highest incidence in the world in that more than 1.13 billion people are hypertensive (Lerman et al., 2019; https://www.who.int/news-room/fact sheets/detail/hypertension). Although the understanding of the pathophysiology of hypertension has advanced considerably in recent years, it is still very difficult to elucidate the causes, as well as to define effective strategies in the treatment of this disease (Hall et al., 2012; Collister et al., 2018). This difficulty is not surprising due to the fact that hypertension is a multifaceted disease resulting from a variety of risk factors (diet, genetics and environment), as well as interactions between them. Adding to this complex nature is the fact that the maintenance of both short and long-term blood pressure is under control of a wide variety of systems such as cardiovascular, neural, endocrine, renal as well as local vascular tissue mediators (Hall et al., 2012; Basting and Lazartigues, 2017). As in many other human diseases, animal models of hypertension have been a valuable tool in understanding its pathogenesis, 
enabling experimentais approaches that would be difficult to reproduce in humans (Lerman et al., 2019). However due to the multifactorial aspects of hypertension, not all available animal models are effective in mimicking the real condition imposed by this disease. One of the most commonly used animal models is mineralocorticoid-induced hypertension in the rat: deoxycorticosteroid acetate (DOCA) which has two variations: 1) DOCA plus added salt (DOCA-salt), which incorporates a high-salt diet (0.6$1 \% \mathrm{NaCl}$ in the drinking water) and 2) DOCA-salt that is accompanied by uninephrectomy (Zicha et al., 1989; Grobe et al., 2011). One of the main advantages of this model is that in both variations, salt sensitivity, a key element for predisposition and development of many cases of hypertension, is present to a similar extent to what occurs in human salt sensitive hypertension (Basting and Lazartigues, 2017).

Previous work in animals non-uninephrectomized animals demonstrated that the development of hypertension occurs more gradually with 28 days of treatment with DOCA resulting in a blood pressure increase of 20 to $30 \mathrm{mmHg}$ (Kandlikar and Fink, 2011). Uninephrectomized animals showed the same pattern of increased blood pressure, but in a shorter period of time after 21 days of treatment with DOCA (Collister et al., 2018). In both cases, animals treated with DOCA demonstrated a typical hypertensive response in the first 7 days of treatment. But non-uninephrectomized animals showed the magnitude of the increase in blood pressure more modest than uninephrectomized rats. We have utilized 21 days of DOCA treatment in uninephrectomized rats because we observe differences between interventional experimental animals and control animals during this shorter time frame. In this DOCA-salt model accompanied by reduced kidney mass (uninephrectomy), chronic hypertension develops in a biphasic response with an initial rise in BP over first 7 days followed by a sustained elevated BP over days 1421 that eventually reaches $125-135 \mathrm{mmHg}$ (Collister et al., 2018).

Since this model is thought to have a neurogenic component mediating the rise in arterial pressure, we sought to determine which area(s) of the brain may be involved in the chronic hypertensive response to DOCA. Therefore, we have previously used this model to investigate the role of the subfornical organ (SFO) and organum vasculosum of the lamina terminalis (OVLT) in the control of arterial blood pressure. Both of these central nuclei are defined as circumventricular organs, hypothalamic structures lacking the blood brain barrier, allowing blood born chemicals such as hormones to gain access to the brain. Previous studies were performed separately in which rats were subjected to electrolytic lesion of either the SFO or OVLT, or respective sham control operation. In the first study, we reported that SFO does not play a significant role in the development of DOCA-salt hypertension as rats with lesions of the SFO responded with a similar chronic hypertensive response to DOCA compared to rats with sham lesions of the SFO. In contrast, lesion of the OLVT significantly attenuated the chronic hypertensive response in rats that consumed a high-salt diet treated with DOCA. Therefore, we concluded the hypertensive response in DOCA-treat rats requires an intact OVLT but not SFO (Osborn et al., 2006; Collister et al., 2018).

In summary, while every animal model has its limitations in comparison to human disease, the DOCAsalt hypertensive model in rats is a reliable animals model that closely mimics many human forms of hypertension in that there are volume-dependent, vascular, and neurogenic components that impact 
both central and peripheral nervous systems (Basting and Lazartigues, 2017, Collister et al., 2018; Lerman et al., 2019).

Finally, in addition to animal models that mimic the physiological changes typical of hypertension, accurate monitoring and recording of blood pressure fluctuations in animal research has helped researchers to unravel several mechanisms involved in the physiology and pathophysiology of diseases such as hypertension and heart failure (Huetteman and Bogie, 2009). Blood pressure and heart rate can be measured directly from the implantation of radio telemetry devices which are considered the 'gold standard' for the measurement of these parameters in intact, conscious laboratory animals during normal behavioral and physiological states (Zhao et al., 2011). These data are collected and recorded without direct human-animal contact during the measurements. Figure 1 shows the basic components of telemetry system used in this protocol.

\section{Basic Components of Telemetry System}

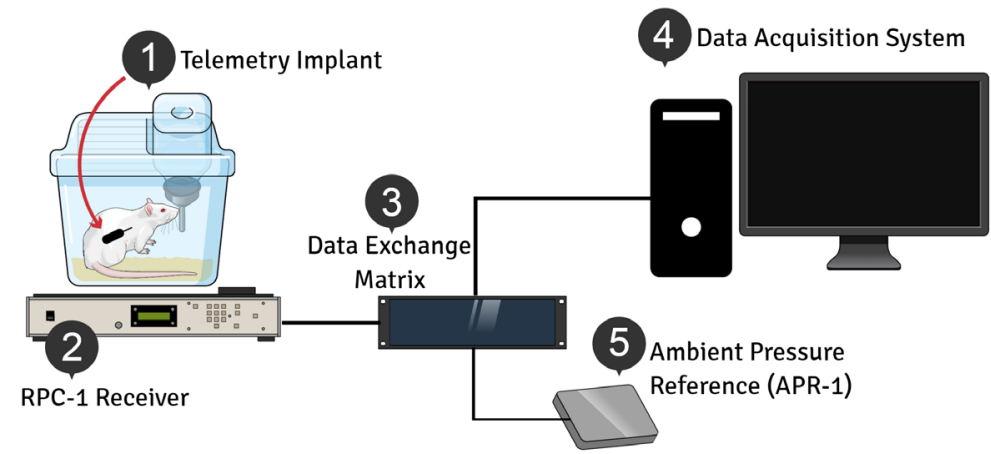

Figure 1. Basic Components of Telemetry System (for more information we recommend to consult Reference 5). 1) Telemetry implant is a device inserted in the rat's abdominal cavity and subsequently senses, measures and transmits the information of mean pressure and heart rate to a nearby receiver via radio telemetry. 2) The RPC-1 receiver detects the transmitted signals from the implant and converts them into a readable form by the computer, and is positioned under or near the animal's cage. 3) Data Exchange Matrix immediately detects the type and serial number of the RPC-1 receiver and forwards this information to the acquisition system. 4) The Data Acquisition System (hardware, software, and computer) removes most corrupt values obtained telemetrically and converts them to common units. In addition, it provides built in routines to plot and analyze acquired data. 5) The Ambient Pressure Reference (APR 1) is needed to measure barometric pressure. Thus, the pressure values detected by the APR-1 are used to correct the pressure measurements obtained from the implant for changes in barometric pressure. 


\section{Materials and Reagents}

1. Needles (Standard Hypodermic Needles, Monoject ${ }^{\top \mathrm{M}}$, catalog numbers: 8881250255 [23G], 8881250149 [21G]), stored at room temperature

2. Sterile drape, $18 "$ x 26" (McKesson Minor Procedure Drape, catalog number: 25-517), stored at room temperature

3. Tape (TimeMed Labeling System, catalog number: 3114), stored at room temperature

4. Syringe (1 ml Tuberculin Syringe Regular Tip) (Monoject ${ }^{\mathrm{TM}}$, catalog number: 8881501400 , stored at room temperature

5. Syringe (3 $\mathrm{ml}$ Luer Lock Syringes) (Monoject ${ }^{\top \mathrm{M}}$, catalog number: 8881513934$)$, stored at room temperature

6. Sterile Gloves (Synthetical Surgical Gloves Powder-Free, Confiderm ${ }^{\circledast}$ SPT), stored at room temperature

7. Gauze sponges (Non-woven Gauze Sponges, 2 in. $\times 2$ in. $[5.08 \mathrm{~cm} \times 5.08 \mathrm{~cm}]$ ) (AVANT GAUZE ${ }^{\circledR}$, catalog number: 25223), stored at room temperature

8. Cotton tip applicators (Non-Sterile Cotton Tipped Applicators, length: 6") (SolonCare ${ }^{\circledR}$, catalog number: 71704), stored at room temperature

9. Polystyrene Weighing Dish (Weigh Dish PS, 1 5/8" x 1 5/8" x 5/16" [Small]) (Fisherbrand ${ }^{\circledR}$, catalog number: 02-204A), stored at room temperature

10. Aluminum foil (Clark Foodservice, catalog number: CF 1851), stored at room temperature

11. Surgical 3-0 Silk (Black Braided Silk, 3-0 USP) (Surgical Specialities, catalog number: SP117), stored at room temperature

12. Surgical staples (Reflex Skin Closure System, Clip Applyer, $7 \mathrm{~mm}$ ) (Agnthos, catalog number: 204-100)

13. Adult male Sprague-Dawley rats (250-275 g; approximately 50 days old, Charles River Laboratory, Wilmington, MA)

14. Deoxycorticosterone acetate, DOCA (Sigma-Aldrich, catalog number: D7000-5G, CAS: 56-47$3)$, stored at room temperature

15. Bag of saline solution (Sodium Chloride $0.9 \%$ Bags for Injection [1,000 ml]) (Baxter, catalog number: 0338-9543-06), stored at room temperature

16. $70 \%$ Isopropyl Alcohol (473 ml) (Medline Industries, catalog number: $53329-800-06)$, stored at room temperature

17. lodine antiseptic solution (Povidone lodine Scrub, $960 \mathrm{ml}$ ) (Prority Care, catalog number: 50009110-32), stored at room temperature

18. Disinfectant soap (Chlorhexidine Gluconate $2 \%$ Solution Antiseptic (Scrub-Stat ${ }^{\mathrm{TM}}$ ECOLAB $^{\circledR}$, catalog number: 47593-463-31), stored at room temperature

19. Heparin 50 units/ml (Heparin Sodium Injection USP, 50 units USP per ml) (B. Braun Medical Inc., catalog number: 0264-9577-10), stored at room temperature 
20. Isoflurane (Rx only, Liquid for inhalation, $250 \mathrm{ml}$ ) (Piramidal Enterprises Limited, catalog number 6679401725), stored at room temperature.

21. Ketoprofen (Ketofen, $100 \mathrm{mg} / \mathrm{ml}$ ) (Zoetis Inc., catalog number: 140269), stored at room temperature

22. Gentamicin sulfate, 200-395 [100 mg/ml] (ANADA, catalog number: 1398563304$)$, stored at room temperature

23. Atropine (Sulfate injection, USP [8 mg/20 ml], catalog number: 0641600601), stored at room temperature

24. Pentobarbitol (Fatal-Plus solution CIl [390 mg/kg, $250 \mathrm{ml}$, RXV CII [2N]), stored at room temperature

25. Buprenorphine hydrochloride (Injection CIII $[0.3 \mathrm{mg} / \mathrm{ml}]$ ) (Buprenex ${ }^{\circledR}$, catalog number: 124960757-5), stored at $20^{\circ} \mathrm{C}$ to $25^{\circ} \mathrm{C}$

26. Lubricant Eye Ointment (Major Pharmaceuticals [3.5 g]) (LubriFresh ${ }^{\mathrm{TM}}$ P.M., catalog number: 0904-5168-38), stored at room temperature

27. Sylgard 184 Silicone Elastomer (Two-part silicone elastomer supplied as flowable liquid, Sylgard $184^{\circledR}[500 \mathrm{~g}]$ ) (Dow Corning, catalog number: 2404019862 ), service range of $-45^{\circ} \mathrm{C}$ to $200{ }^{\circ} \mathrm{C}$

28. Standard Rat Chow (2018 18\% Protein Rodent Diet, Teklad Global Diets ${ }^{\circledR}$ ) (Envigo, catalog number: 44220), stored at room temperature

29. $0.1 \% \mathrm{NaCl}$ powdered food (Mature Rodent Diet with $0.1 \%$ added Sodium Cloride, Open Source Diets $^{\text {TM }}$, Research Diets) (New Brunswick, catalog number: D17020M), stored at $4{ }^{\circ} \mathrm{C}$

30. Sodium Chloride, $\mathrm{NaCl}$ (Sodium Chloride Certified ACS Crystaline, $500 \mathrm{~g}$ ) (Fisher Chemical, catalog number: S271-500), stored at room temperature

31. Fiber Patch (PA-C40 cellulose patch, Data Sciences International, St. Paul, MN)

32. VetBond surgical adhesive (Tissue Adhesive, $3 \mathrm{ml}$ Bottles w/MSDS) (3M Vetbond ${ }^{\mathrm{TM}}$, catalog number: $1469 \mathrm{SB})$, stored at room temperature

33. $0.9 \% \mathrm{NaCl}$ drinking solution (see Recipes)

\section{Equipment}

1. Instruments (Figure 2)

Micro dissecting retractor (A1 and A4)

Long hemostatic forceps (A2)

Needle holder (A3)

Curved hemostatic forceps (A5)

Micro dissecting straight little scissors (A6)

Surgical scissors (A7, A8 and A14)

Auto-clip applier for $9 \mathrm{~mm}$ (A9)

Scalpel with scalpel blade number 10 (A10)

Scalpel handle (A11) 
Needle support (A12)

Micro aneurism clip (A13)

Forceps (A15 and A17)

Medium hemostatic forceps (A16)

Dissecting forceps with teeth (A18)

Micro dissecting Forceps (A19)

Small Spatula (B1)

Weighing Dish (B2)

A.

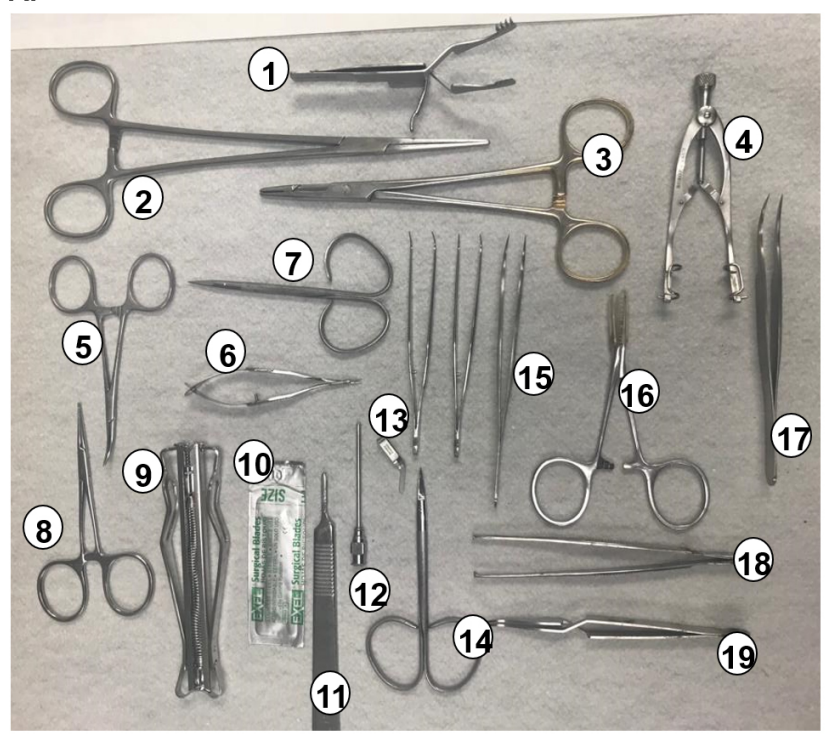

B.

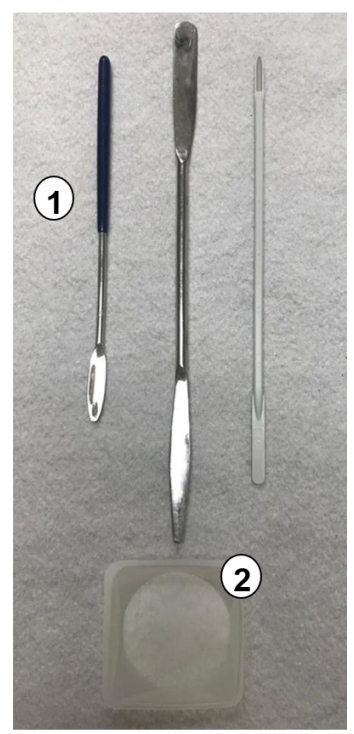

Figure 2. Instruments used in surgical procedures (A) and preparation of DOCA pellets (B)

2. Supplemental heating (Pad Heating, 18" x 26" [46 cm x $66 \mathrm{~cm}]$ ) (MultPad, Stryker, catalog number: TP26E), stored at room temperature

3. Clipper for hair removal (Oster ${ }^{\circledR}$ Golden $A 5^{\circledR}$, Detachable Blade, 120 Volts AC, 45 Watts; [size 40])

4. Metabolic cages (Nalgene Metabolic Cage) (Ancare, catalog number: 650-0100)

5. Single Cage Stand (Ancare, Bellmore, NY. For more information: www.ancare.com)

6. Vertical Metabolic Cage Rack (Ancare, Bellmore, NY. For more information: www.ancare.com)

7. Isoflurane vaporizer (Vapomatic Anesthetic vaporizer, SKU: 20010, NY) (Vapomatic ${ }^{\mathrm{TM}}$, catalog number: 2200)

8. Veterinary Anesthesia Machine (Am Bickford Anesthesia Machine, catalog number: 61020; SKU: 61020B, NY)

9. Rat Induction chamber (Induction Chamber, 1 Liter 3.75"W x 4.50"D x 3.75"H Designed for a single mouse or small rat) (VetEquip ${ }^{\circledR}$, catalog number: 941443 )

10. Heat Therapy Pump (T/Pump ${ }^{\circledR}$, Stryker Medical, model: TP 700) 
11. PA-C40 transmitter (TA11PA-C40, Data Sciences International, catalog number: 270-0040-008), stored at room temperature

12. AM Radio (FM/AM, DC: 3V R6 Size AA x 2 Batt or Equiv, Sony ${ }^{\circledR}$, model: ICF: S10MK2)

13. Telemetry receiver (RPC-I, DSI ${ }^{\mathrm{TM}}$, catalog number: 272-6001)

14. Data Exchange MatrixTM (DSI ${ }^{\mathrm{TM}}$, St Paul, Minnesota, USA)

15. Ambient Pressure Reference (DSI ${ }^{\mathrm{TM}}$, model: APR-1)

16. IBM computer

\section{Software}

1. Dataquest IV software (DSI ${ }^{\mathrm{TM}}, \underline{w w w}$.dataquest.io)

2. Microsoft Excel

3. Sigma Plot (Sigma Plot 9.0, The Technical Graphing Standard, SYSTAL, www.systal.com)

4. Mind the graph (www.mindthegraph.com)

\section{Procedure}

A. Unilateral nephrectomy and transmitter implantation

Instruments and other materials used for surgeries must be sterile. There are several methods of sterilization. We recommend for this protocol steam sterilization (autoclaving) of all instruments. Rats should be weighed and doses of anesthetics and medications given according to individual body weight.

B. Anesthesia and Pre-Surgical procedures

1. Fast the rats the night before the surgical procedures (approximately $8 \mathrm{~h}$ before).

2. Place the rats in an induction chamber in which isoflurane is delivered in $100 \%$ oxygen. The induction must start at $0.5 \%$ of isoflurane every $5 \mathrm{~min}$, until the gas induction reaches $5 \%$ (for more details see Reference 9).

3. Apply eye ointment to the eyes for lubrication.

4. When the animals have reached a surgical plane of anesthesia, remove from the chamber and placed on the heating pad (make sure it has circulating warm water) and secure the rats with tape and position them in dorsal recumbency.

5. Pull the rat's tongue to the side of the mouth to maintain an open airway.

6. Maintain a state of deep anesthesia by using a nose cone administering isoflurane at $2 \%$ in oxygen.

7. Provide pre-operative doses of atropine $(0.5 \mathrm{mg} / \mathrm{kg}$; intramuscular), gentamicin $(6 \mathrm{mg} / \mathrm{kg}$; subcutaneously) and ketoprofen (2.5 mg/Kg; subcutaneously).

8. Maintain all rats on a heating pad during surgery and postoperative recovery. 
9. Once fully anesthetized animals, characterized by lack of response to clamping pressure with fingers applied to the distal tail, shave the rats (clipping) in the areas needed for the surgical procedure (Figure 3 ) and scrub the surgical site 3 times with iodine antiseptic solution, then $70 \%$ alcohol as recommended by Institutional Animal Care and Use Committee (IACUC) and Research Animal Resources (RAR).

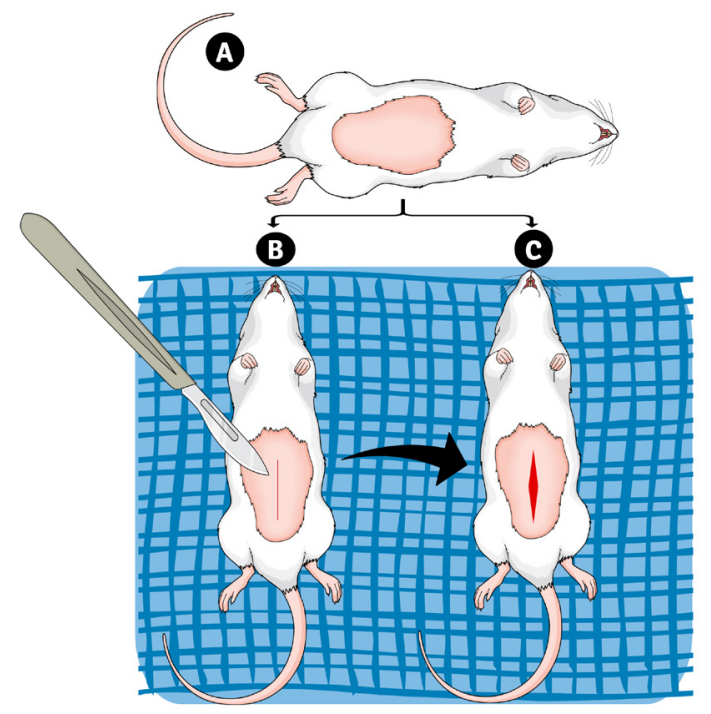

Figure 3. Schematic of rat's preparation for the surgical procedures. Area to be shaved (A). Incision (B) and opening of the abdominal cavity (C).

Note: Perform both surgeries at the same time on each rat.

10. Using sterile gloves, drape the surgical area to prevent contamination of the disinfected site, cute a hole in the drape. In the case of multiple surgeries, it is recommended no more than 6 animals may be done with the one set of instruments, and between one and another clean and sterile instruments. In addition, new sterile gloves must be donned.

11. Approximately $10 \mathrm{~min}$ before the surgical procedure, open transmitter package, record the serial number and ensure the device has been identified with the animal in which it will be implanted.

C. Surgeries (Figure 4)

Unilateral nephrectomy surgery (Zwemer, 1999 provides more detailed step by step instructions of this procedure): 


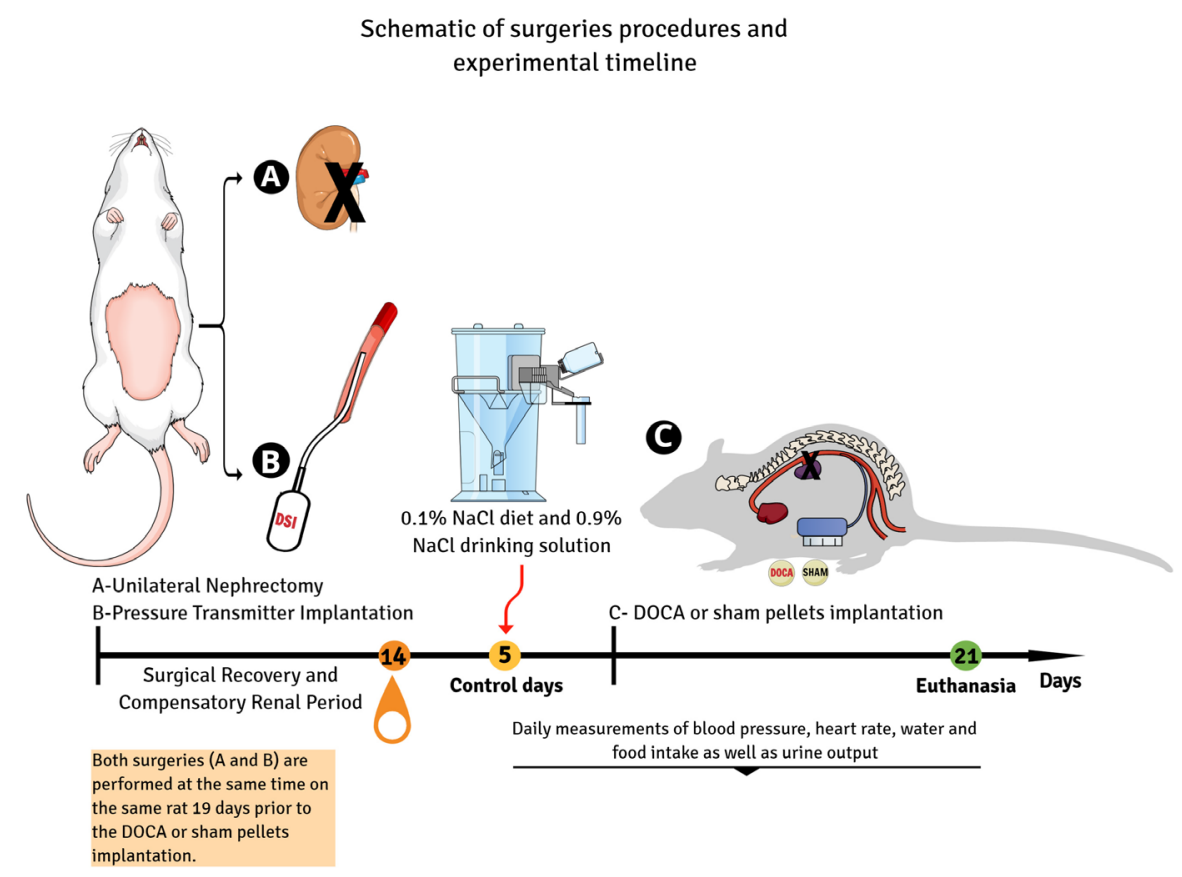

Figure 4. Schematic of surgeries procedures and experimental timeline. Unilateral nephrectomy (A). Pressure transmitter implantation (B). Perform both surgeries at the same time in each rat approximately 19 days before the DOCA or sham pellet implantation. After the surgical procedures provide 14 days of recovery before the next procedure, allowing for compensatory renal hypertrophy associated with the unilateral nephrectomy. During this recovery period, the animals are housed in normal cages and have access to standard rat chow and water ad libitum. After the 2 weeks of recovery, transfer all rats to individual metabolic cages and provide $0.1 \% \mathrm{NaCl}$ diet and ad libitum $0.9 \% \mathrm{NaCl}$ drinking solution. Daily, during 5 control days, measure water and food intake as well as urine output, blood pressure and heart rate. After this control period perform surgery $(C)$ for subcutaneous implantation of DOCA or sham pellets. During 21 days after DOCA or sham pellet implantation perform daily measurements of blood pressure, heart rate, food and water intake and urine output. At the end of the experiment, euthanize the rats deeply with a lethal dose of pentobarbital sodium.

1. Make a 4-6 cm midline incision using a sterile number 10 scalpel blade through the skin gently dissecting from the abdominal wall.

2. Cut abdominal muscles along the midline linea alba using sharp scissors to expose the contents of the abdomen (stomach, small bowel, large bowel, spleen, pancreas, and mesenteric arteries and veins).

3. For better visualization, the kidneys can be exposed by gently pulling on the viscera and releasing them out of the abdomen and pushing them to the right side of the animal.

4. Identify and isolate the renal blood supply using two ligatures. Tie off artery and vein together at the hilus of the kidney using sterile 3-0 silk suture. The right ureter is connected to the renal 
hilus and should be tied off as well. Cut the vessels and ureter using iris scissors (Figure 2, instrument A8) close to the hilus.

5. Carefully isolate the fat and connective tissue surrounding the right kidney to avoid damaging the adjacent adrenal gland and remove the kidney.

Transmitter implantation (Figure 5; Reference 4 and Cesarovic et al., 2011 provides more detailed step by step instructions of this procedure):

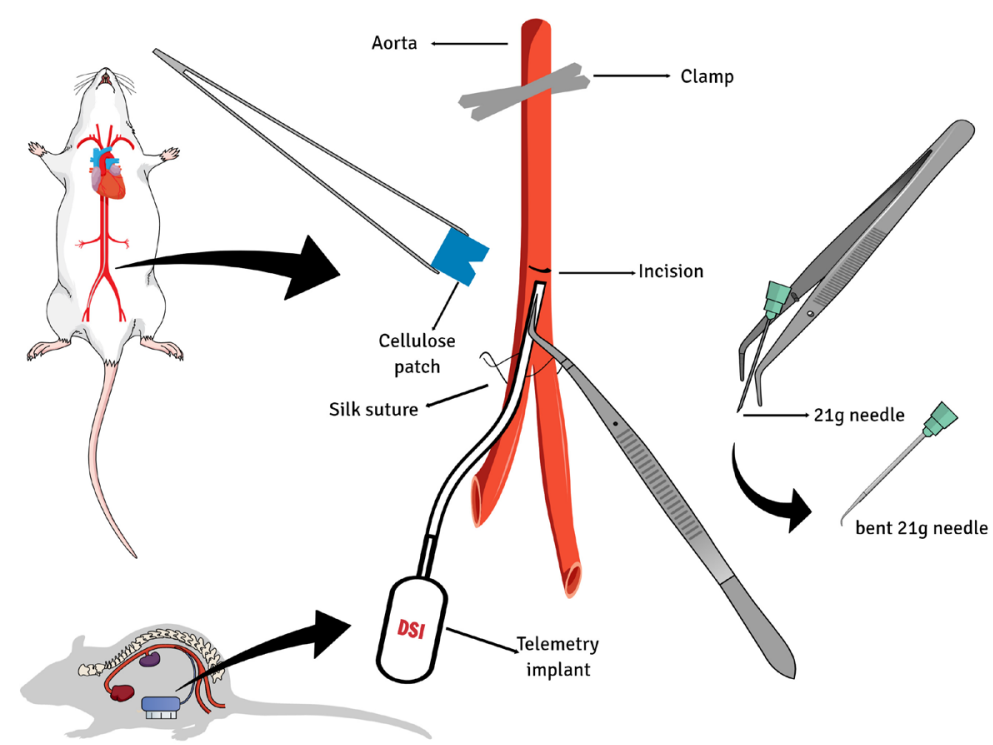

Figure 5. Schematic of transmitter implantation. The catheter is placed into the abdominals aorta between the renal arteries and the iliac bifurcation. The telemetry device is anchored to the abdominal wall closure (modified by Reference 4).

6. Using moistened cotton applicators, gently part the intestines to allow visualization of the descending aorta located along the dorsal body wall. Retract the intestines using moistened gauze sponges.

7. Next, the descending aorta must be visualized. Using sterile cotton applicators, dissect carefully and completely the surrounding fat and connective tissue from the aorta.

8. Before the catheterization, clear excess tissue from the ventral surface of aorta. This ensures good hemostasis.

9. Once everything is ready for the implantation, clamp the aorta just distal to the renal arteries (Figure 2, instrument A13) and puncture with a bent 21-gauge needle distal to the clamp (just proximal to the distal aortic bifurcation).

Note: At this point, the time for catheter placement is limited due to potential ischemia caused by clamping.

10. Insert the tip of the telemetric device catheter (Data Science International, St. Paul, MN) into the abdominal aorta and guide upstream (using forceps A17), cranially approximately $1 \mathrm{~cm}$. Apply 
a small drop of tissue adhesive (Vetbond surgical adhesive) and a surgical patch (Data Science International) to secure the catheter and obtain hemostasis.

11. Wait approximately $30 \mathrm{~s}$ for the adhesive to bond and remove the clamp.

12. The transmitter placement in the aorta can be verified after the clamp is removed because the device transmits an AM radio frequency and can be heard on small AM radio situated near the body of the transmitter (Video 1).

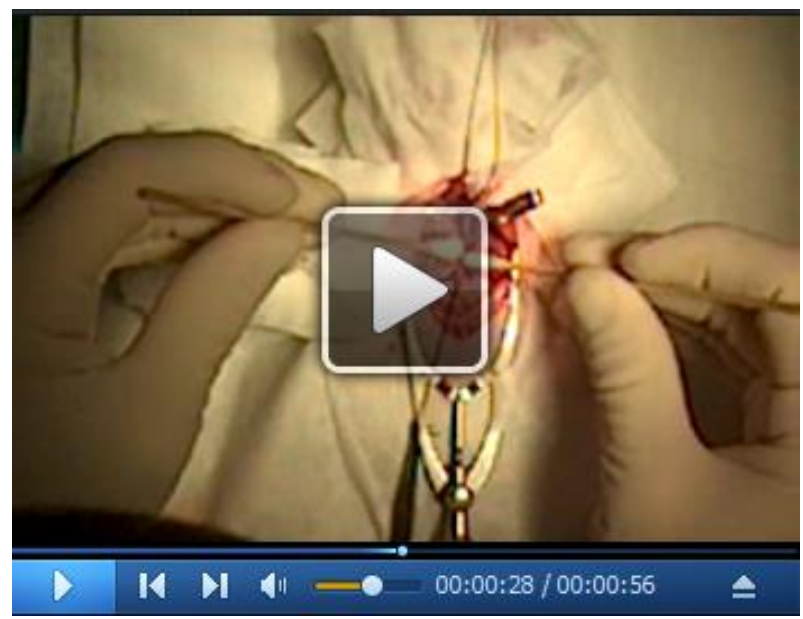

Video 1. The transmitter placement in the aorta

13. Place the body of the telemetric device in the abdominal cavity and suture it in the abdominal musculature using sterile 3-0 silk suture.

14. Gently replace the abdominal contents in rats and close the laparotomy in layers (close abdominal musculature and skin individually) using sterile 3-0 silk suture.

15. Upon completion of surgery, place the animals in individual cages, with half of the cage on the heating pad until rats are awake.

16. Using a small scissor remove the suture and staples approximately 7-10 after the surgeries.

\section{Post-surgical treatment:}

17. Provide buprenorphine for 3 days Bis In Die (B.I.D; 0.05-0.1 mg/kg; subcutaneously) and access to normal rat chow and water ad libitum.

18. Monitor the rats daily for BARG signs (bright, alert, responsive and grooming) of good health. If the animals do not show BARG signs it is recommended to contact the veterinarians to assess their condition and if they are viable for use in the experiment.

19. Give approximately 14 days to recover in standard cages before the next procedure, allowing for compensatory renal hypertrophy associated with the unilateral nephrectomy.

Metabolic cages (Figure 6): assessment of daily water and food intake, and urine output 


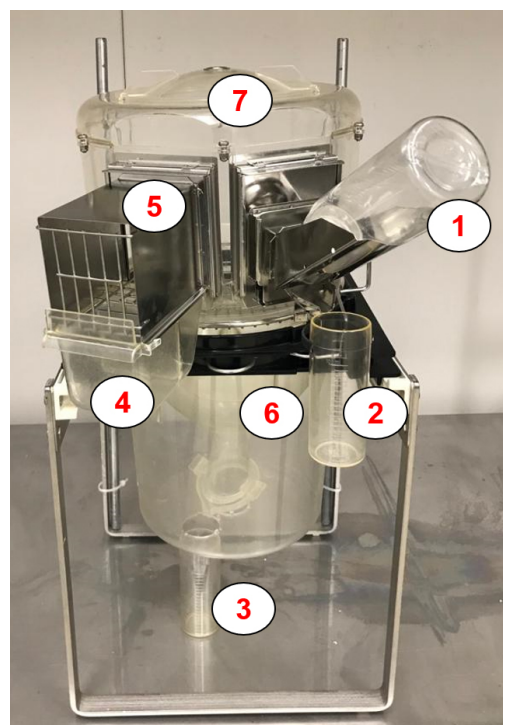

Figure 6. Individual metabolic cage (Nalgene Nunc, Rochester NY). Metabolic cages for rodents are an important tool for studies in which it is necessary to measure water/food intake, feces and urine output. In general, all components are dismountable and washable. The containers for water and urine (1 and 2 ) and urine (3) are cylindrical plastic tubes that facilitate accurate measurements of consumption and output, respectively. The food is stored in a plastic drawer (4) that easily slides without disturbing the animal. Feeder Chamber (5), located outside cage. A collection funnel and separating cone (6) provide complete separation of feces and urine (For more information we recommend to consult Ancare website).

20. After 2 weeks of recovery, keep all rats housed individually in metabolic cages (Nalgene Nunc, Rochester, NY), provide $0.1 \% \mathrm{NaCl}$ diet and ad libitum $0.9 \% \mathrm{NaCl}$ drinking solution.

21. During this period measure 24-h water and food intake. These measures are obtained by subtracting the amount of water/food offered by the amount of water/food remaining on the following day at the same time.

22. To measure the urine output, note the volume contained in the specific cylinder located under the cage.

23. Calculate sodium intake as the sum of the sodium received from saline intake $(0.154 \mathrm{meq} / \mathrm{ml})$ plus the product of the food intake and the sodium content of the food $(0.1 \% \mathrm{NaCl}, 3570.0175$ $\mathrm{mmol} / \mathrm{g}$ ).

24. Calculate the daily sodium and water balances as the difference between intake and urinary excretion of sodium and water, respectively.

D. DOCA or sham pellets implant surgery

Make the DOCA and sham silicone implants at least $72 \mathrm{~h}$ prior to surgical implantation.

\section{Pellet preparation}

1. Weigh $100 \mathrm{mg}$ of DOCA into one small weigh boat (see Figure 2; B2). Repeat for as many needed within the week. 
2. Draw up $2 \mathrm{ml}$ of Part A of Sylgard 184 Silicone in $3 \mathrm{ml}$ syringe, and add to the weigh boat with the pre-weighed DOCA. Mix thoroughly will spatula for $\sim 30-45 \mathrm{~s}$. Be sure to break up any small clumps, as homogeneity is extremely important to the quality of the pellet*

Note: *This is a critical step in the preparation of pellets. If these are not well homogenized absorption rate is not consistent and the results may be confounded due to variability.

3. Draw up $0.2 \mathrm{ml}$ of curing agent from the silicone kit in a $1 \mathrm{ml}$ syringe, and add the $0.2 \mathrm{ml}$ to the silicone mixture. Mix thoroughly again for $\sim 30-45 \mathrm{~s}$.

4. Cover with pre-labeled aluminum foil (i.e., $100 \mathrm{mg} \mathrm{DOCA}$ and date), and set aside to cure for $24 \mathrm{~h}$ at room temperature.

5. Let the DOCA implants cure at room temperature for $24 \mathrm{~h}$ and then refrigerate at $4{ }^{\circ} \mathrm{C}$ until the day of the surgical implantation.

6. The sham pellets are prepared as a vehicle identically as described above without the addition of the DOCA.

Implantation: This procedure must be performed after 5 control days after the 2 weeks recovery period (see Figure 4).

7. Prior to surgery, use a sterile scalpel to slice DOCA pellet into $\sim 1-2 \mathrm{~mm}^{3}$ pieces, and transfer contents to large, ethanol-cleaned spatula.

8. After anesthetizing all rats and providing pre-surgical treatments and disinfection procedures as described above, implant DOCA or sham pellets.

9. Shave and clean the left flank. Make a skin incision $(\sim 3-4 \mathrm{~cm})$, and use a mayo scissors to form a subcutaneous pocket.

10. Insert spatula into opening, and push the pellet (one per rat) into pocket.

11. Close surgical site with staples or suture.

12. As describe above, after surgery keep the animals in individual cages, half of the cage must be kept on the heating pad until they return from anesthesia.

Note: Pellets will likely stick or pile up, so be sure to flatten and disperse with finger pressure once the surgical opening is closed.

13. Return rats to their home cages upon recovery and provide analgesia to the rats with ketoprofen ( $2.5 \mathrm{mg} / \mathrm{kg} \mathrm{IP}$ ) once daily for 3 days post-surgery. Continue to provide ad libitum access to their $0.1 \% \mathrm{NaCl}$ powdered food and $0.9 \% \mathrm{NaCl}$ drinking solution.

14. Maintain the rats individually housed in respective metabolic cage, which are positioned on the receiver (set up DSI monitoring equipment [Figure 1], $500 \mathrm{~Hz}$ for $10 \mathrm{~s} / \mathrm{min}$ ) and make daily measurements of mean arterial pressure (MAP) and heart rate (HR), food and water intake and urine output for 21 days after DOCA implantation.

Note: After placing the animals in the individual metabolic cages located on the receivers, it is important to wait 15-25 min for the cardiac parameters to return to baseline levels. 


\section{Euthanasia}

15. At the end of the experiment, euthanize the rats deeply with a lethal dose of pentobarbital sodium (65 mg/kg, intraperitoneal).

\section{Data analysis}

Twenty-four-hour averages were collected for BP, HR, sodium intake, sodium output, water intake and urine output. Results were reported as means $\pm S$.E. Two-way ANOVA combined with a StudentNewman-Keuls test was used for group comparisons. Differences were considered significant at $P$ $<0.05$ (Collister et al., 2018).

\section{$\underline{\text { Recipes }}$}

1. $0.9 \% \mathrm{NaCl}$ drinking solution
a. Dissolve $9 \mathrm{~g}$ of $\mathrm{NaCl}$ in $1,000 \mathrm{ml}$ of distilled water
b. Stir until thoroughly dissolved
c. Store the solution at room temperature

\section{Acknowledgments}

The original studies that supported this paper, "Effect of subfornical organ lesion on the development of mineralocorticoid-salt hypertension" and "Lesion of the OVLT markedly attenuates chronic DOCAsalt hypertension in rats", were funded by a grant from the National Heart Lung and Blood Institute of the National Institutes of Health (HL64178) and University of Minnesota Grant-In-Aid 107426 to J.P.Collister.

\section{Competing interests}

The authors declare no conflicts of interest (financial or any otherwise) involved in this article.

\section{Ethics}

All procedures described were approved and monitored by the Institutional Animal Care and Use Committee (IACUC protocol number 1509-32991A - Role of OVLT in DOCA hypertension approved between 12/21/2015-12/20/2018). 


\section{References}

1. Basting, T. and Lazartigues, E. (2017). DOCA-Salt Hypertension: an Update. Curr Hypertens Rep 19(4): 32.

2. Cesarovic, N., Jirkof, P., Rettich, A. and Arras, M. (2011). Implantation of radiotelemetry transmitters yielding data on ECG, heart rate, core body temperature and activity in free-moving laboratory mice. J Vis $\operatorname{Exp}(57)$ : e3260.

3. Collister, J. P., Nahey, D. B., Hartson, R., Wiedmeyer, C. E., Banek, C. T. and Osborn, J. W. (2018). Lesion of the OVLT markedly attenuates chronic DOCA-salt hypertension in rats. Am J Physiol Regul Integr Comp Physiol 315(3): R568-R575.

4. DSI ${ }^{\circledR}$ DATA Sciences International. "PA Device Surgical Manual". (Printed for custumers)

5. DSI ${ }^{\circledR}$ DATA Sciences International. "Telemetry Information Guide". (Printed for custumers)

6. Grobe, J. L., Buehrer, B. A., Hilzendeger, A. M., Liu, X., Davis, D. R., Xu, D. and Sigmund, C. D. (2011). Angiotensinergic signaling in the brain mediates metabolic effects of deoxycorticosterone (DOCA)-salt in C57 mice. Hypertension 57(3): 600-607.

7. Hall, J. E., Granger, J. P., do Carmo, J. M., da Silva, A. A., Dubinion, J., George, E., Hamza, S., Speed, J. and Hall, M. E. (2012). Hypertension: physiology and pathophysiology. Compr Physiol 2(4): 2393-2442.

8. Huetteman, D. A. and Bogie, H. (2009). Direct blood pressure monitoring in laboratory rodents via implantable radio telemetry. Methods $\mathrm{Mol}$ Biol 573: 57-73.

9. JoVE Science Education Database. (2020). Anesthesia Induction and Maintenance. Lab Animal Research. JoVE, Cambridge, MA.

10. Kandlikar, S. S. and Fink, G. D. (2011). Splanchnic sympathetic nerves in the development of mild DOCA-salt hypertension. Am J Physiol Heart Circ Physiol 301(5): H1965-1973.

11. Lerman, L. O., Kurtz, T. W., Touyz, R. M., Ellison, D. H., Chade, A. R., Crowley, S. D., Mattson, D. L., Mullins, J. J., Osborn, J., Eirin, A., Reckelhoff, J. F., Iadecola, C. and Coffman, T. M. (2019). Animal Models of Hypertension: A Scientific Statement From the American Heart Association. Hypertension 73(6): e87-e120.

12. Osborn, J. W., Jacob, F., Hendel, M., Collister, J. P., Clark, L. and Guzman, P. A. (2006). Effect of subfornical organ lesion on the development of mineralocorticoid-salt hypertension. Brain Res 1109(1): 74-82.

13. Zicha, J., Kunes, J., Lebl, M., Pohlova, I., Slaninova, J. and Jelinek, J. (1989). Antidiuretic and pressor actions of vasopressin in age-dependent DOCA-salt hypertension. Am J Physiol 256(1 Pt 2): R138-145.

14. Zhao, X., Ho, D., Gao, S., Hong, C., Vatner, D. E. and Vatner, S. F. (2011). Arterial Pressure Monitoring in Mice. Curr Protoc Mouse Biol 1: 105-122.

15. Zwemer, C. F. (1999). Unilateral nephrectomy in the rat as a teaching model of renal function. Am J Physiol 276(6 Pt 2): S79-85. 